\title{
Tratamento endovascular embolizante de pseudoaneurisma em rim transplantado
}

\section{Endovascular embolization treatment of pseudoaneurism in transplanted kidney}

Victor Hugo Lauro Soares ${ }^{1}$. José Edison de Andrade Filho ${ }^{1}$. Frederico Augusto de Carvalho Linhares Filho ${ }^{1}$. Gabriela Perina Bernhardt².

1 Hospital Universitário Walter Cantídio (HUWC), Fortaleza, Ceará, Brasil. 2 Hospital Geral de Fortaleza (HGF), Fortaleza, Ceará, Brasil.

\section{RESUMO}

O pseudoaneurisma de artéria renal é uma complicação vascular que pode ocorrer após procedimentos percutâneos, nefrectomia, traumas penetrantes e traumas contusos, este de difícil ocorrência. Os sinais e sintomas são inespecíficos e podem variar com cada paciente, sendo a hematúria o sintoma mais comum. Com isso, faz-se necessário um bom entendimento do histórico médico do paciente para suspeição clínica. A tomografia computadorizada e o ecodoppler podem ser usados como auxílio ao diagnóstico. Neste relato, apresentamos o caso de um paciente submetido à transplante renal com enxertia em vasos ilíacos à esquerda, evoluiu com piora de função renal sendo submetido a biópsia renal. O quadro progrediu com queda do hematócrito e piora da função renal, sendo submetido a ultrassom com Doppler de rim transplantado que evidenciou formação anecóica pulsátil. A tomografia mostrou extravasamento de contraste no parênquima do enxerto renal. Foi optado, então, por tratamento endovascular de pseudoaneurisma em enxerto renal com molas. O paciente evoluiu no pós-operatório com resolução da dor em fossa ilíaca esquerda, e melhora de função renal.

Palavras-chave: Transplante de rim. Falso aneurisma. Procedimentos endovasculares.

\section{ABSTRACT}

Renal artery pseudoaneurysm is a vascular complication that may occur after percutaneous procedures, nephrectomy, penetrating trauma and blunt trauma, which is difficult to perform. Signs and symptoms are non-specific and may vary with each patient, with hematuria being the most common symptom. With this, it is necessary to have a good understanding of the patient's medical history for clinical suspicion. Computed tomography and Doppler can be used as a diagnostic aid. In this report, we present the case of a patient who underwent renal transplantation with graft in the iliac vessels to the left, and progressed with worsening renal function undergoing renal biopsy. The condition progressed with a decrease in hematocrit and worsening renal function, being submitted to ultrasound with transplanted kidney Doppler showing anechoic pulsatile formation. Tomography showed contrast extravasation in the renal graft parenchyma. It was then chosen by endovascular treatment of pseudoaneurysm in renal graft with springs. The patient evolved postoperatively with resolution of left iliac fossa pain, and improved renal function.

Keywords: Kidney transplantation. False aneurysm. Endovascular procedures.

Autor correspondente: Victor Hugo Lauro Soares, Rua Jornalista Nertam Macêdo, 100, Cocó, Fortaleza, Ceará. CEP: 60192-450. Telefone: +55 85 99922-9285. E-mail: soaresvhl@gmail.com

Conflito de interesses: Não há qualquer conflito de interesses por parte de qualquer um dos autores.

Recebido em: 01 Out 2018; Revisado em: 09 Jun 2019; Aceito em: 14 Ago 2019. 


\section{INTRODUÇÃO}

O Pseudoaneurisma daArtéria Renal(PAR) é uma complicação vascular rara, a qual está associada etiologicamente com nefrectomia parcial, procedimentos percutâneos, biópsia renal, trauma penetrante e, mais raramente, trauma contuso. ${ }^{1-3}$ Dentre as causas, as traumáticas ou iatrogênicas são as mais frequentes. ${ }^{4}$ Para o diagnóstico dessa lesão vascular, existem vários exames complementares disponíveis como a ultrassonografia com Doppler, arteriografia, angiotomografia, cintilografia renal e a ressonância magnética.,3 Para o tratamento, pode-se citar seis modalidades: expectante, cirúrgico, compressão, injeção de salina, embolização com mola, injeção de trombina, sendo a embolização a indicada para aqueles de menores dimensões em pacientes estáveis hemodinamicamente.

\section{RELATO DE CASO}

Paciente masculino, 49 anos, submetido à transplante renal em dezembro de 2017, tendo sido o implante do enxerto renal realizado em vasos ilíacos à esquerda. Em janeiro de 2018, evoluiu com piora de função renal, com creatinina de $3,2 \mathrm{mg} / \mathrm{dl}$, sendo submetido a biópsia renal no dia 30 do mesmo mês. Após o procedimento, paciente persistiu com aumento das escórias nitrogenadas, com creatinina atingindo $4,7 \mathrm{mg} / \mathrm{dl}$, redução acentuada de diurese para $350 \mathrm{ml} / 24 \mathrm{hs}$ e dor em fossa ilíaca esquerda, associado a queda de hemoglobina de $8,5 \mathrm{mg} / \mathrm{dl}$ para $7 \mathrm{mg} / \mathrm{dl}$.

Foi submetido a Ultrassom (USG) com Doppler de rim transplantado que evidenciou formação anecoica pulsátil, exibindo padrão ao Doppler de fluxo arterial e com sinal de Yin-yang no terço médio anterior do enxerto de $3,8 \times 2,0 \mathrm{~cm}$, sendo caracterizado pseudoaneurisma intrarrenal.

Para o melhor estudo da imagem evidenciada no USG, foi realizada tomografia computadorizada com contraste que evidenciou extravasamento de contraste no parênquima do enxerto renal conforme Figura 1.

Figura 1. Tomografia computadorizada evidenciando extravasamento de contraste intrarrenal.

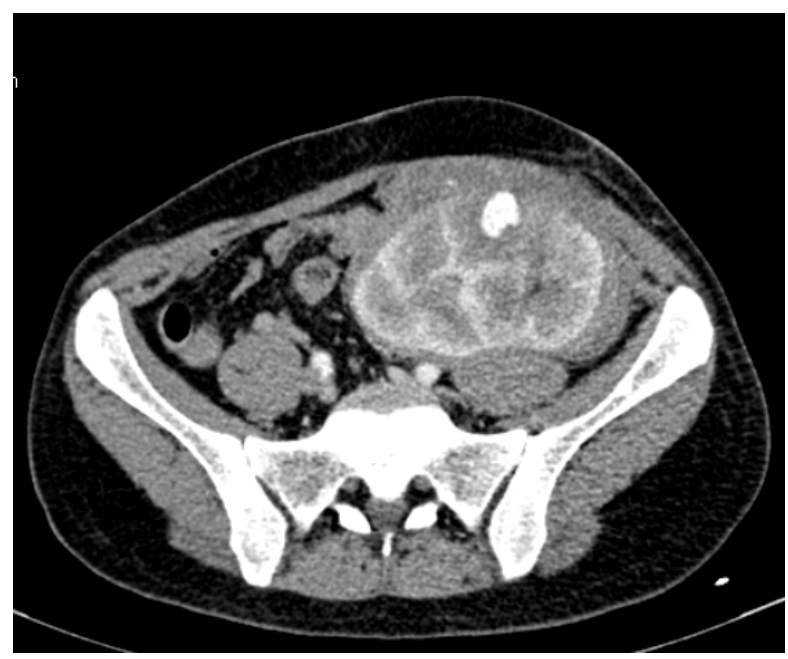

Após o diagnóstico tomográfico, foi optado por tratamento endovascular de pseudoaneurisma em enxerto renal. Foi realizada a punção da artéria femoral esquerda, seguido de aposição de introdutor 5 french (fr) e angiografia sob subtração digital. Evidenciado pseudoaneurisma intrarrenal com fluxo, proveniente de ramo arterial terciário (Figura 2). Com o auxílio de um guia 0,014 polegadas e micro cateter 2,6 Fr foi cateterizado o ramo nutridor do pseudoaneurisma, obtendo-se melhor definição da lesão (Figura 3).

Figura 2. Angiografia de artéria renal evidenciando pseudoaneurisma.

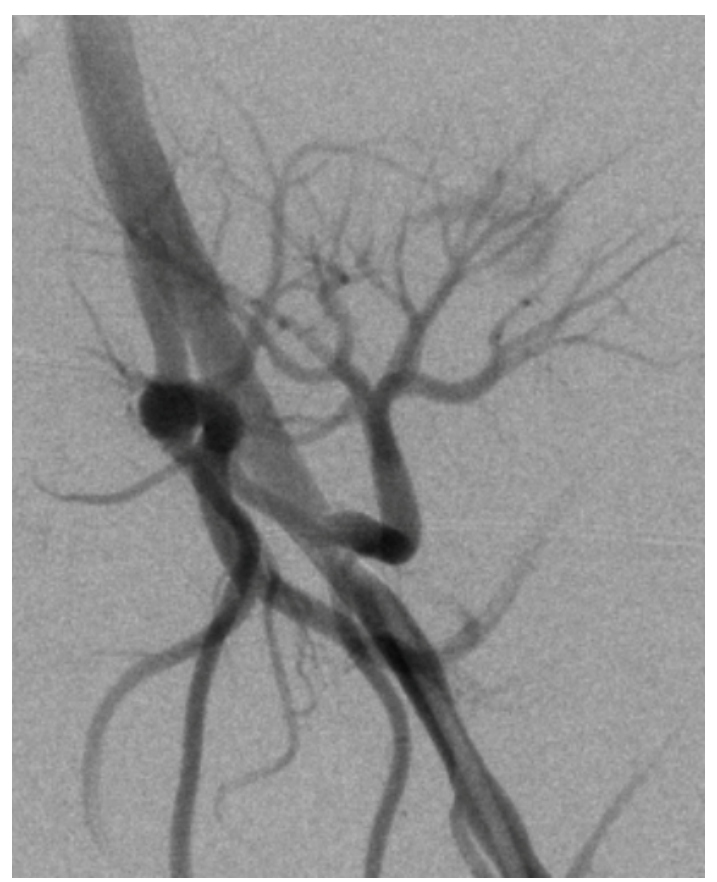

Figura 3. Angiografia com cateterização seletiva de pseudoaneurisma.

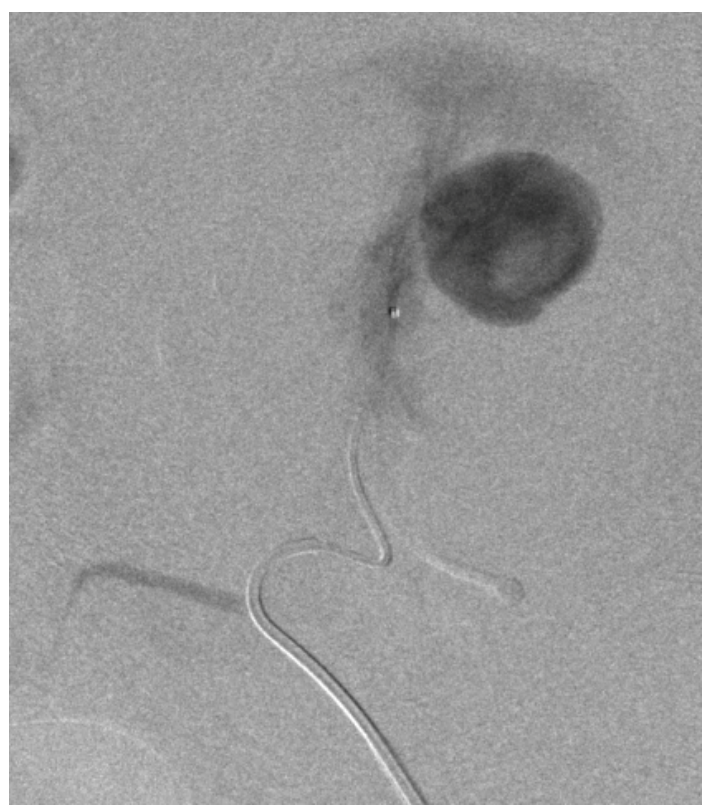


Optou-se pela embolização com molas de liberação não controlada do pseudoaneurisma por serem as disponíveis no serviço. Foi obtido sucesso no tratamento conforme a aquisição angiográfica após a embolização (Figura 4).

O paciente evoluiu no pós-operatório com resolução da dor em fossa ilíaca esquerda, estabilização da hemoglobina em $7,4 \mathrm{mg} / \mathrm{dl}$ e melhora de função renal com nova creatinina de $3,2 \mathrm{mg} / \mathrm{dL}$ sete dias após o procedimento. O paciente foi acompanhado no ambulatório da cirurgia vascular 30 dias após o procedimento mantendo nível de creatinina em $3,1 \mathrm{mg} / \mathrm{dl}$.

Figura 4. Resultado final após embolização com molas.

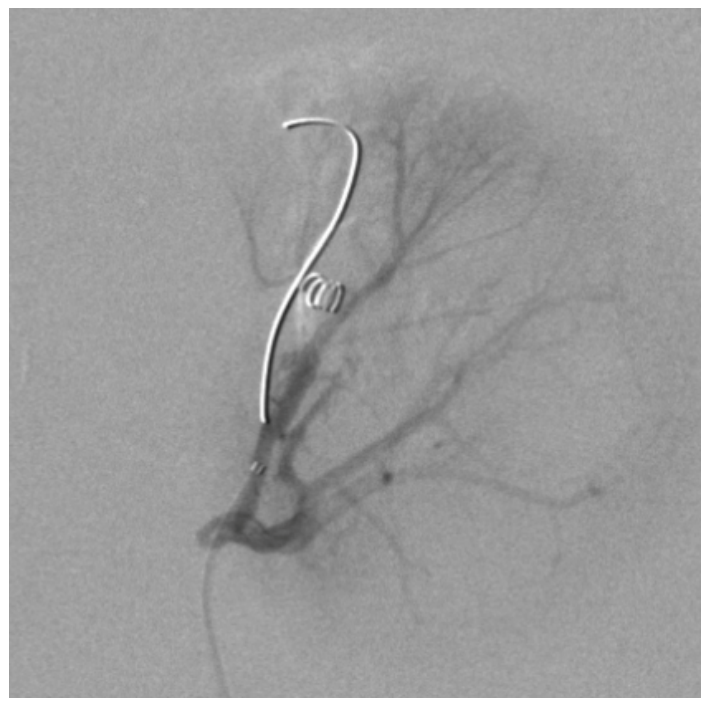

\section{DISCUSSÃO}

Os pseudoaneurismas se formam em locais de lesão arterial onde a hemorragia estava contida nos tecidos não vasculares adjacentes. Eles são mais propensos a ocorrer quando apenas um lado da parede arterial está lesionado, enquanto que a transecção completa de uma artéria geralmente não resulta em formação de pseudoaneurisma. ${ }^{1}$ Qualquer lesão arterial penetrante pode resultar em formação de pseudoaneurismas, incluindo cirurgia reconstrutiva vascular, punção inadvertida por usuários de drogas intravenosa, trauma fechado, fixação interna ortopédica, bem como intervenções percutâneas ou vasculares. ${ }^{2}$

A incidência de pseudoaneurisma iatrogênico aumentou devido à crescente prevalência de procedimentos intervencionistas periféricos, cateterismo cardíaco transarterial e biópsias guiadas por imagens. Eles ocorrem em 0,1\%-0,2\% dos angiogramas de diagnóstico e 3,5\%-5,5\% dos procedimentos de intervenção. ${ }^{3}$ No caso de biópsia guiada por imagem, o tamanho da agulha da biópsia foi considerado proporcional à taxa de complicações. ${ }^{4}$

A incidência de pseudoaneurisma foi relatada como maior com o uso de trombolíticos, medicamentos antiplaquetários, anticoagulantes, ${ }^{5}$ bem como em pacientes com baixa contagem de plaquetas e/ou disfunção plaquetária. ${ }^{6}$ As opções de tratamento de pseudoaneurismas incluem reparo de compressão guiada por ultrassom, tratamentos percutâneos minimamente invasivos (injeção de trombina, embolização de bobina e inserção de stents cobertos) ou reparação cirúrgica. ${ }^{5}$ O reparo de compressão guiada por ultrassom é uma opção de tratamento econômica, porém, que consome muito tempo para pseudoaneurismas superficiais. Desvantagens desta técnica incluem desconforto do paciente e alta taxa de recorrência em pacientes com anticoagulação e aumento da taxa de falha em pseudoaneurismas com diâmetros acima de $3 \mathrm{~cm} .{ }^{7,8}$ Em nosso estudo de caso, o tamanho grande e os locais profundos dos pseudoaneurismas excluíram a utilização desta técnica.

Os princípios de tratamento para pseudoaneurismas em comparação com aneurismas verdadeiros diferem muito. Embora o reparo cirúrgico tenha sido ao mesmo tempo um pilar do tratamento, outras opções de gerenciamento menos invasivas são agora preferidas em pacientes selecionados adequadamente. ${ }^{9}$ A compressão guiada por ultrassom pode ser um método eficaz de tratamento.

A ultrassonografia duplex é usada para identificar o pseudoaneurisma e a quantidade adequada de pressão é aplicada para eliminar o fluxo através do pertuito do pseudoaneurisma, mantendo o fluxo através do lúmen arterial nativo. A pressão excessiva pode causar a complicação indesejada de trombose da própria artéria. A pressão deve ser aplicada durante pelo menos 10 minutos com reavaliação para o fluxo perseverante através do pseudoaneurisma. Se o fluxo continuar, ciclos adicionais de 20 minutos de compressão devem ser executados até ocorrer uma trombose bem-sucedida. Esta abordagem de tratamento pode ser limitada em pacientes que sofrem de dor extrema ou em pessoas obesas. A cronicidade da lesão é diretamente proporcional à quantidade de tempo necessário para a trombose completa. É importante notar que a ruptura do pseudoaneurisma resultante da força excessiva é uma complicação séria que pode ocorrer. ${ }^{10}$ Assim, esta estratégia de gerenciamento deve ser limitada a lesões localizadas em áreas de acesso anatômico fácil.

Outras opções incluem injeção guiada por ultrassom de agentes como a trombina que estimulam a coagulação do pseudoaneurisma. ${ }^{10,11}$ As terapias guiadas por ultrassom têm a vantagem de evitar meios de contraste, os perigos da irradiação e as complicações do cateterismo angiográfico. ${ }^{12} \mathrm{~A}$ injeção de trombina para tratamento do pseudoaneurisma é uma excelente opção terapêutica para os pseudoaneurismas (PA) superficiais, e aqueles que apresentam um colo longo e de pequeno diâmetro possuem custo inferior comparado com tratamento endovascular, pois pode ser realizado guiado por ultrassom, sem necessidade dos custos inerentes ao uso de sala de hemodinâmica e acesso arterial e sem as complicações relacionadas com a punção arterial. Porém, PA profundos e com colos de diâmetros maiores e trajetos curtos apresentam maior risco de complicações como oclusão arterial aguda por vazamento da trombina para o vaso sadio. ${ }^{9} \mathrm{~A}$ terapêutica endovascular constitui uma excelente opção no tratamento dos PAR por serem menos invasivos, com 
baixos índices de complicações quando comparados com a cirurgia convencional aberta. ${ }^{10}$ Entre as opções de tratamento endovascular, a embolização percutânea superseletiva com molas constitui uma alternativa terapêutica para o tratamento de pseudoaneurisma de baixo risco e morbidade por reduzir o território vascular em risco de infarto e apresenta boas taxas de resolução dos pseudoaneurisma. Complicações no tratamento com molas podem decorrer da liberação inadvertida em outros vasos não implicados nos PA o que pode levar a oclusão arterial

\section{REFERÊNCIAS}

1 Hernando Arteche A, Alpuente Roman C, Martin del Toro R, Pérez-Piqueras Gómez A, Sainz González F, Cabrera Cabrera JA. Pseudoaneurisma de artéria renal principal associado a fistula cecal tras nefrectomia radical: caso clínico y revisión de la literatura. Sanid Mil. 2014;70:95-7.

2 Octavio Castillo C, Jorge Díaz M, Eduardo Caffarena A, Manuel Díaz C, Jorge Hoyos M. Pseudoaneurisma arteria renal postnefrectomía parcial: tratamiento exitoso con embolización selectiva. Rev Chil Cir. 2006;58(5):382-5.

3 Melo RA, Pires MJ, Silva LM, Segovia AB. Giant renal artery pseudoaneurysm 18 months after blunt trauma. J Vasc Bras. 2013;12:159-62.

4 Galego SJ, Barbato H, Correa JA, Kafejian O, Polimanti AC, Fürst $\mathrm{RV}$, et al. Pseudo-aneurisma de artéria renal pós-endopielotomia retrógrada: relato de caso e revisão de literatura. J Vasc Br. 2004;3(3):285-7.

5 Pastorín R, Rodríguez N, Polo AM, Vicente JM, Luján M. Posttraumatic giant renal pseudoaneurysm. Emerg Radiol. 2007;14(2):117-21.

6 Yang HK, Koh ES, Shin SJ, Chung S. Incidental renal artery pseudoaneurysm after percutaneous native renal biopsy. BMJ Case Rep. $2013 ; 2013$. e consequentemente infartos teciduais. As molas de liberação semi-controlada e controladas constituem uma alternativa mais segura para o tratamento dos PA por permitirem melhor precisão na sua liberação, porém, de custo financeiro maior.

A terapia endovascular é segura, minimamente invasiva e constitui uma alternativa eficaz de tratamento das complicações vasculares como o PAR em pacientes submetidos a biópsia renal, inclusive de rins transplantados.

7 Dou C, Jin Y, Sun S, Zhou A, Chen X. Interventional treatment of post-biopsy renal artery pseudoaneurysm in a child: case report and literature review. Zhonghua Er Ke Za Zhi. 2015;53(10):775-8.

8 Albani JM, Novick AC. Renal artery pseudoaneurysm after partial nephrectomy: three case reports and a literature review. Urology. 2003;62(2):227-31.

9 Hirsch AT, Haskal ZJ, Hertzer NR, Bakal CW, Creager CW, Halperin JL, et al. Peripheral arterial disease: ACC/AHA 2005 guidelines for the management of patients with peripheral arterial disease (lower extremity, renal, mesenteric, and abdominal aortic). J Am Coll Cardiol. 2006;47:1239-1312.

10 Koliskor B, Mccoul ED, Goldstein NA, Weedon J, Jackson A, Goldsmith AJ. Retrospective review of detachable neurovascular microcoils in endovascular treatment of renal pseudoaneurysms and AV fistulae. Jour Vasc Interv Rad. 2016;27(3):S271.

11 Kang SS, Labropoulos N, Mansour MA, Michelini M, Filliung D, Baubly MP, et al. Expanded indications for ultrasound-guided thrombin injection of pseudoaneurysms. J Vasc Surg. 2000;31:28998 .

12 Sakr MA, Desouki SE, Hegab SE. Direct percutaneous embolization of renal pseudoaneurysm. J Endourol. 2009;23:8758.

\section{Como citar:}

Soares VH, Andrade JE Filho, Linhares FA Filho, Bernhardt GP. Tratamento endovascular embolizante de pseudoaneurisma em rim transplantado. Rev Med UFC. 2020 jan-mar;60(1):59-62. 